\title{
Portal Venous-Phase CT of the Liver in Patients without Chronic Liver Damage: Does Portal-Inflow Tracking Improve Enhancement and Image Quality?
}

\author{
Masayuki Kanematsu*, Haruo Watanabe, Hiroshi Kondo, Satoshi Goshima, \\ Hiroshi Kawada, Yoshifumi Noda \\ Department of Radiology, Gifu University Hospital, Gifu, Japan \\ Email: masa_gif@yahoo.co.jp
}

Received June 7, 2013; revised July 7, 2013; accepted July 15, 2013

Copyright (C) 2013 Masayuki Kanematsu et al. This is an open access article distributed under the Creative Commons Attribution License, which permits unrestricted use, distribution, and reproduction in any medium, provided the original work is properly cited.

\begin{abstract}
Purpose: This study was undertaken to determine if portal-inflow bolus tracking outperforms aortic bolus tracking with respect to the image quality of contrast-enhanced portal venous-phase CT of the liver in patients without chronic liver damage. Materials and Methods: Contrast-enhanced CT of the liver was performed in 132 consecutive patients without chronic liver damage. Patients were prospectively assigned to three protocols: Protocol A - a portal venous-phase scan delay of 6 seconds after superior mesenteric venous (SMV) enhancement increased by $70 \mathrm{HU}$ or 14 seconds after SMV enhancement was visually confirmed, and Protocols B and C-40 and 50 seconds, respectively, after abdominal aortic enhancement increased by $100 \mathrm{HU}$. Enhancement $(\Delta \mathrm{HU})$ of abdominal aorta, portal trunk, and liver parenchyma and diagnostic acceptability were assessed. Results: $\triangle \mathrm{HU}$ of aorta was higher for protocol A than for protocols $\mathrm{B}$ and $\mathrm{C}$ $(P<0.05)$, whereas $\Delta \mathrm{HU}$ of portal trunk was higher for protocol B than for protocols $\mathrm{A}$ and $\mathrm{C}(P<0.05)$. $\Delta \mathrm{HUs}$ of liver were similar in three protocols. No difference was found between diagnostic acceptabilities of three protocols. Conclusion: Portal-inflow bolus tracking did not outperform aortic tracking in terms of optimization of portal venous-phase $\mathrm{CT}$ in patients without chronic liver damage.
\end{abstract}

Keywords: CT; Liver; Contrast Enhancement; Bolus Tracking

\section{Introduction}

Usefulness of contrast-enhanced CT for the diagnosis of hepatic diseases is widely recognized and the technique is employed at many centers. Furthermore, temporally resolved multi-phasic CT scanning after the intravenous bolus injection of contrast material is a crucial for the detection and characterization of focal hepatic lesions [1-5]. In particular, the acquisition of optimal portal venousphase images is essential for the diagnosis of hyper- or hypovascular liver metastases [3-5].

Several researchers have described methods of optimizing scan delays after contrast injection, in particular, for the hepatic arterial-dominant phase imaging, with the use of bolus-tracking [6], test-bolus imaging [7], and fixed injection duration [8] techniques. Bolus-tracking is widely employed in many centers to optimize scan pro-

\footnotetext{
${ }^{*}$ Corresponding author.
}

tocols of contrast-enhanced CT of the liver, including the acquisition of hepatic arterial-dominant phase images and subsequently of portal venous-phase images.

Portal venous-phase images play an important role in the detection of malignant hepatic tumors by maximizing the tumor-to-liver contrast, and in the characterization of focal hepatic lesions by demonstrating washout of malignant hepatic tumors [9] or the peripheral paddling of cavernous hemangiomas $[2,10]$. Nowadays, because the total scan time for the entire liver has reduced to as little as 2 seconds, we wondered whether a dedicated technique could allow scanning of the whole liver and capture the most intense enhancement of liver parenchyma during the portal venous phase. The purpose of this study was to determine if portal-inflow tracking outperforms the widely employed aortic bolus tracking for the optimization of contrast-enhanced portal venous-phase CT of the liver in patients without chronic liver damage. A literature search failed to unearth any reports on this topic. 


\section{Materials and Methods}

\subsection{Patients}

This HIPAA-compliant study had institutional review board approval and all patients provided written informed consent. During a recent 5-month period, 176 consecutive patients with a known malignancy and without known chronic liver disease due to viral, alcoholic, autoimmune, or cryptogenic hepatitis underwent abdominopelvic contrast-enhanced $\mathrm{CT}$ for a preoperative work-up or a post-therapeutic survey. Of these, 44 patients were excluded because of; prior abdominal surgery that could significantly alter the portal venous blood flow dynamics $(n=38)$, diffuse fatty liver $(n=2)$, and numerous liver metastases $(n=4)$. The remaining $132 \mathrm{pa}-$ tients (88 men and 44 women; age range, 19 - 91 years; mean age, 62.1 years) constituted the study population. The primary malignancies in these 132 patients were; rectal $(n=35)$, gastric $(n=29)$, colon $(n=28)$, breast $(n$ $=10)$, uterine cervical $(n=5)$, endometrial $(n=3)$, esophageal $(n=3)$, ovarian $(n=3)$, prostate $(n=2)$, pancreatic $(n=2)$, renal cell $(n=1)$, bile duct $(n=1)$, appendiceal $(n=1)$, pulmonary $(n=1)$, urinary bladder $(n=1)$ or testicular $(n=1)$ carcinoma, or gastrointestinal stromal tumor of the stomach $(n=2)$, malignant lymphoma of the cervical lymph nodes $(n=2)$, uterine leiomyosarcoma $(n$ $=1)$, and Paget's disease of the scrotum $(n=1)$.

\subsection{Contrast Material Injection and Scan Protocols}

A 16-detector CT scanner (Lightspeed 16; GE Healthcare, Milwaukee, WI) with a fixed tube voltage of $120 \mathrm{kVp}$ and an automatic tube current modulation program (3D mA Modulation; GE Healthcare) was used. Other CT parameters were as follows: collimation, $1.25 \mathrm{~mm}$; detector configuration, 16 detectors with a $1.25-\mathrm{mm}$ section thickness $(16 \times 1.25 \mathrm{~mm})$; table feed, $27.5 \mathrm{~mm}$ per rotation; pitch, 1.37; craniocaudal scan range, $45-50 \mathrm{~cm}$; $32-\mathrm{cm}$ field of view; 0.5 -second gantry rotation time; and scan acquisition time, 8.8 - 9.7 seconds. All transverse $\mathrm{CT}$ images were reconstructed at section thickness of 5 $\mathrm{mm}$ by using a standard reconstruction algorithm.

After the acquisition of unenhanced images, a bolus-tracking program (Smart Prep; GE Medical Systems) was used to determine the optimal time to initiate diagnostic portal venous-phase scanning following the administration of contrast medium. All patients were administered non-ionic iodinated contrast material containing $300 \mathrm{mg}$ of iodine per milliliter warmed to body temperature at a dose of $2 \mathrm{~mL}$ per kilograms injected intravenously over 30 seconds using a commercially available power injector through a 21-gauge plastic catheter, which was typically placed in either an antecubital vein or a radial vein. The fractional dose was $20 \mathrm{mg}$ iodine $/ \mathrm{kg} / \mathrm{sec}$ in all patients.

Patients were prospectively randomized to three protocol groups, using a random-number table, as follows: Protocol A-the portal venous-phase scan was started 6 seconds after enhancement of the superior mesenteric vein (SMV) trunk increased by $70 \mathrm{HU}$ or 14 seconds after the SMV enhancement was visually confirmed, and Protocols B and C-40 and 50 seconds, respectively, after the abdominal aortic enhancement increased by 100 HU. For protocol A, CT number measurement in the SMV occasionally failed due to respiratory motion. Hence, we determined the empiric scan delay by visually confirming SMV enhancement prior to initiating the portal venous-phase scan. The default empiric scan delay was determined to be 14 seconds, which was obtained by adding 8 seconds to the 6 seconds, because the mean time in seconds from the initiation of contrast inflow in the SMV to a CT number increase by $70 \mathrm{HU}$ was determined to be $8.1 \pm 3.7$ (mean $\pm 1 \mathrm{SD}$ ) seconds during our preliminary evaluation of 40 patients (unpublished our data).

The region of interest cursor for bolus tracking was placed in the SMV trunk or in the aorta just above the diaphragmatic dome. Real-time low-dose $(120 \mathrm{kVp}, 50$ $\mathrm{mA}$ ) serial monitoring scans were started 30 (protocol A) or 10 (protocols $\mathrm{B}$ and $\mathrm{C}$ ) seconds after initiating the contrast injection. The time in seconds from initiation of contrast injection to diagnostic scan initiation was recorded in all patients.

\subsection{Quantitative Image Analysis}

A radiologist (H. W.) with 5 years of post-training experience at interpreting body CT images measured mean CT numbers and determined standard deviations in the abdominal aorta, portal vein trunk, and liver on a commercially available DICOM viewer. Measurements were performed on unenhanced and portal venous-phase axial images. CT numbers of livers were measured in the left lateral, right anterior, and right posterior segments devoid of blood vessels, bile ducts, focal hepatic lesions, calcifications, and artifacts, and the numbers obtained were averaged. Quantitative degrees of contrast enhancement are expressed as $\mathrm{CT}$ numbers increases from unenhanced to contrast-enhanced axial images $(\Delta \mathrm{HU})$.

\subsection{Qualitative Image Analysis}

Two radiologists (H. Kondo and S. G.) with 13 and 10 years of post-training experience of interpreting body CT images, respectively, who were unaware of patient clinical information and CT imaging parameters prospectively and independently reviewed CT images. Each radiologist first graded images alone, and subsequently, consensus grades were reached by discussion. 
The radiologists independently graded portal venousphases CT images separately for diagnostic acceptability using a five-point scale (grade $1=$ unacceptable, grade 2 = suboptimal, grade $3=$ acceptable, grade $4=$ good, grade $5=$ excellent). A grade was awarded to each patient after reviewing all images. Grade 5 was given when the image quality (soft tissue contrast, sharpness of tissue interfaces, lesion conspicuity, and paucity of image degradation caused by streaking noise or beam-hardening artifacts) was deemed superb; grade 3 when image quality was fair and did not hamper image interpretation; and grade 1 when image quality was considerably poor enough to hampered image interpretation. Grades 4 and 2 were defined as being intermediate between grades 5 and 3 and grades 1 and 3 , respectively.

\subsection{Statistical Analysis}

Statistical analyses were performed using commercially available software (SPSS, version 17; SPSS, Chicago, Ill). For quantitative measurements, one-way analysis of variances (ANOVA) was performed to compare background factors (patient age and body weight) and mean $\Delta \mathrm{HUs}$ of the abdominal aorta, portal trunk, and liver between the three protocols. When a statistically significant intergroup difference was found by ANOVA, pair-wise comparisons were performed using the Mann-Whitney test with Bonferroni correction, and a stricter $p$ value criterion of $<0.017$ was considered significant. The KruskalWallis test was used to compare qualitative grades. When a significant difference was found between the three protocols, pairwise comparisons were performed using the Mann-Whitney test with Bonferroni correction, and again a stricter $p$ value criterion $<0.017$ was considered significant.

\section{Results}

\subsection{Patient Background Factors}

The protocol A, B, and C groups consisted of 46, 43, and 43 patients, respectively. No significant difference was found between any two groups in terms of age or body weight (Table 1). Medians times from initiations of contrast injections to initiations of diagnostic scans were 47, 56 , and 67 seconds for the three protocols, respectively (Figure 1).

\subsection{Quantitative Image Analysis}

Means values and the 1 SDs of the $\Delta H U$ s of the abdominal aortas, portal veins, and livers for the three protocols are summarized in Table 2, and medians and $\Delta \mathrm{HU}$ variabilities are summarized in Figure 2. The mean $\Delta \mathrm{HU}$ of the abdominal aorta was higher for protocol A than for protocols B and $\mathrm{C}(P<0.05)$, and the mean $\Delta \mathrm{HU}$ of the
Table 1. Patient age and body weight in the three protocols.

\begin{tabular}{cccc}
\hline & $\begin{array}{c}\text { Protocol A } \\
(n=46)\end{array}$ & $\begin{array}{c}\text { Protocol B } \\
(n=43)\end{array}$ & $\begin{array}{c}\text { Protocol C } \\
(n=43)\end{array}$ \\
\hline Age $(\mathrm{y})$ & $61.3 \pm 11.6$ & $63.1 \pm 10.7$ & $61.9 \pm 14.3$ \\
Body weight $(\mathrm{kg})$ & $60.1 \pm 10.5$ & $57.1 \pm 9.8$ & $55.7 \pm 11.6$ \\
\hline
\end{tabular}

No significant difference in age $(P=0.77)$ and body weight $(P=0.15)$ was found between any two protocols.

Table 2. Contrast enhancement of the abdominal aorta, portal vein, and liver in the three protocols.

\begin{tabular}{cccc}
\hline & $\begin{array}{c}\text { Protocol A } \\
(n=46)\end{array}$ & $\begin{array}{c}\text { Protocol B } \\
(n=43)\end{array}$ & $\begin{array}{c}\text { Protocol C } \\
(n=43)\end{array}$ \\
\hline $\begin{array}{c}\text { Abdominal } \\
\text { Aorta }\end{array}$ & $130.0 \pm 17.6^{*}$ & $120.4 \pm 18.6$ & $116.6 \pm 16.6$ \\
Portal vein & $135.8 \pm 14.7$ & $144.7 \pm 21.3^{* *}$ & $128.7 \pm 15.5$ \\
Liver & $58.2 \pm 9.2$ & $56.2 \pm 8.8$ & $54.8 \pm 9.3$ \\
\hline
\end{tabular}

Note: Numbers are mean $\Delta \mathrm{HU} \pm 1 \mathrm{SD} .{ }^{*}$ Value was significantly greater than those in protocols $\mathrm{B}$ and $\mathrm{C}(P<0.05){ }^{* * *}$ Value was significantly greater than those in protocols $\mathrm{A}$ and $\mathrm{C}(P<0.05)$.

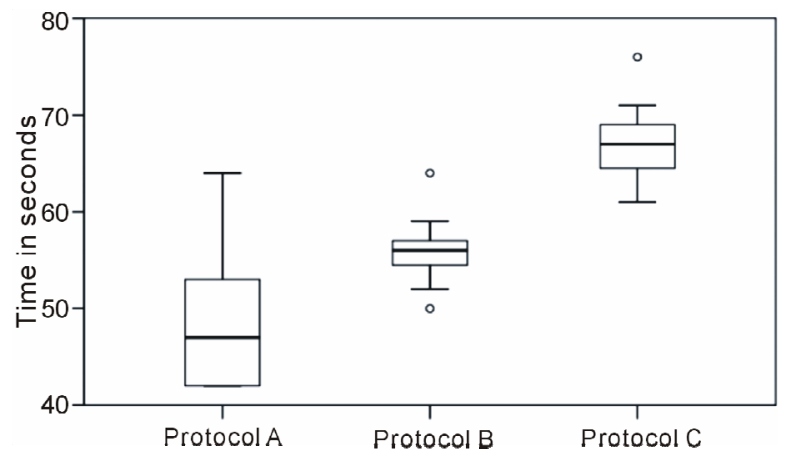

Figure 1. Box plot showing time in seconds from initiation of intravenous contrast injection to initiation of portal venous diagnostic scan. Median values were 47, 56, and 67 seconds for protocols $A, B$, and C, respectively. Boundary of boxes closest to zero indicates 25 th percentile, line within boxes indicates median, and boundary of boxes farthest from zero indicates 75 th percentile. Error bars indicate smallest and largest values within 1.5 box lengths of 25th and 75 th percentiles. Outliers are represented as individual points.

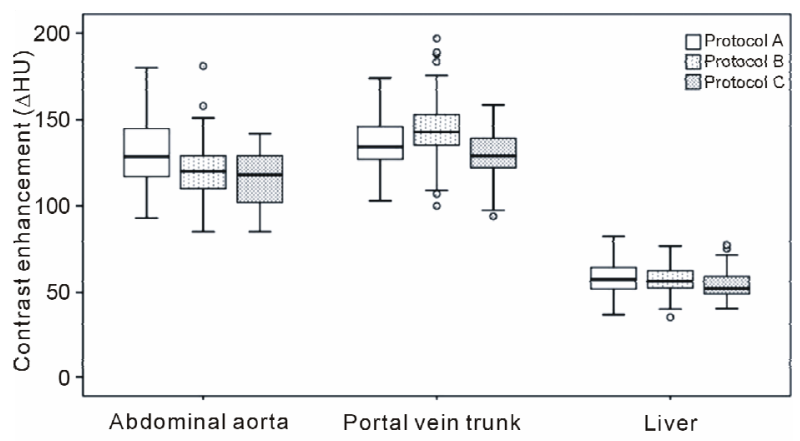

Figure 2. Box plot showing increased contrast enhancement in $\mathrm{HU}$ from unenhanced to portal venous-phase images for abdominal aorta, portal vein trunk, and liver. Note same definitions of symbols as those in Figure 1. 
portal trunk was higher for protocol B than for protocols $\mathrm{A}$ and $\mathrm{C}(P<0.05)$. On the other hand, the mean liver $\Delta$ HUs in the three groups were comparable.

\subsection{Qualitative Image Analysis}

The mean grades for diagnostic acceptability were $4.30 \pm$ $0.70,3.93 \pm 0.73$, and $4.19 \pm 3.88$ for protocols $\mathrm{A}, \mathrm{B}$, and $\mathrm{C}$, respectively (Figures 3 and 4 ). No difference was found between the three protocols.

\section{Discussion}

The median time from initiating contrast injection to initiating a diagnostic scan with SMV tracking (protocol A) was 47 seconds, and this was rather shorter than 56 and 67 seconds required for aortic tracking (protocols $\mathrm{A}$ and $\mathrm{B}$, respectively). Furthermore, the variability of these durations was greater with SMV tracking. The greater variability observed when employing SMV tracking was expected, because these durations were strongly affected by circulation time variations of the portal venous system

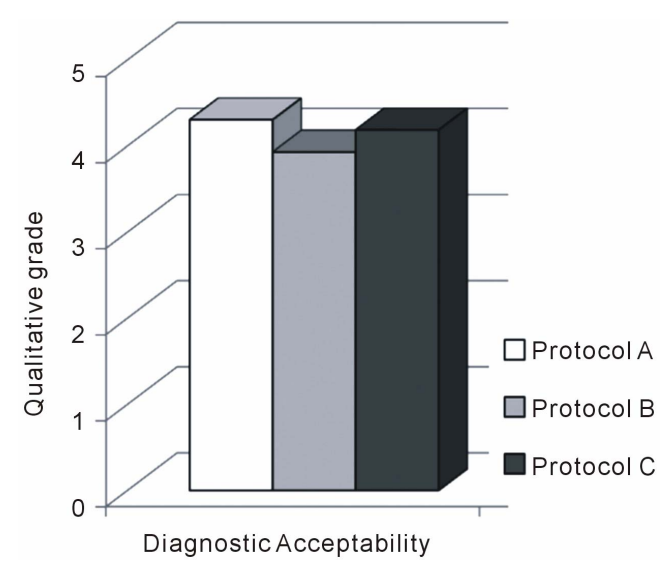

Figure 3. Bar chart showing diagnostic acceptability grades. No difference was found between three protocols.

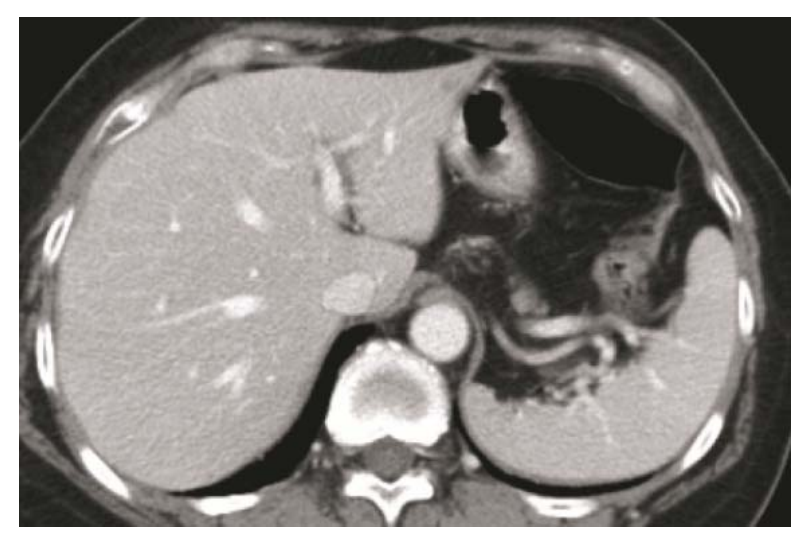

Figure 4. Portal venous-phase CT image in 56-year-old woman weighing 63 kilogram examined using SMV tracking (protocol A). Intensity of hepatic enhancement and image quality are acceptable in this case. and systemic circulation. Although we set the delay time from bolus detection in the SMV to maximize hepatic contrast enhancement based on the results of our preliminary study, the actual time from initiation of contrast injection to portal venous-phase diagnostic scan was shortest for protocol A. However, had we even extended the delay time with SMV tracking by for example nine seconds, so that median of the duration was the same as that of protocol B, liver enhancement might have been similar to that observed in the present study.

Despite the difference of no less than 20 seconds in median times from initiation of contrast injection to scan start between SMV and aortic tracking methods, no intergroup difference in hepatic enhancement was observed, which suggested that durations of peak hepatic enhancement in the portal venous phase was sustained fairly long. Therefore, in contrast to scan timing optimization for the hepatic arterial-dominant phase, the scan time window for the portal venous phase was rather wide, and hence strict scan timing optimization might not be necessary for portal venous-phase imaging.

Livers were enhanced on average by 54 - 58 HU during the portal venous phase for all three protocols and no significant difference was observed. HU increases of livers in the present study are consistent with those of a previous study [11], in which it was shown that maximum hepatic enhancement in the portal venous phase was linearly correlated with the iodine dosage $(\mathrm{mg} / \mathrm{kg}$ of body weight). In this previous study, an iodine dose of $520 \mathrm{mg} / \mathrm{kg}$ yielded a hepatic enhancement by $50 \mathrm{HU}$ [11].

In the present study, the standard deviations of hepatic enhancement for all three protocols lay in the range 8.8 9.3 HU, and no difference was found between the protocols. Furthermore, qualitative image qualities (based on diagnostic acceptability) were similar for the three protocols. These observations also concur well with those of the previous study [11], in which maximum hepatic enhancement was found to be strongly correlated with iodine dosage $(\mathrm{mg} / \mathrm{kg})$ as long as portal venous-phase images were obtained during the fairly long, peak liver enhancement.

When we undertook this study, we also sought to determine whether portal venous tracking allows variabilities in the timing of contrast material portal inflow to be corrected and allows stably capture of maximum hepatic enhancement using a rapid CT scanner that enables a whole liver scan to be conducted within seconds. However, SMV tracking was not found to produce stronger hepatic enhancement despite the somewhat more complex procedure required as compared with aortic tracking.

In the present study, enhancement of the portal vein was significantly greater for aortic tracking with a 40second scan delay (protocol B). One possible explanation 
for the significant difference in portal venous enhancement observed despite no difference in hepatic enhancement in the portal venous phase is that in the protocol B the scan timing was most consistent with the timing of the portal inflow of dense contrast material and the timing was somewhat premature for the hepatic parenchymal imaging, although no differences in hepatic enhancement were found.

This study has some limitations that warrant consideration. First, this study was conducted on a limited cohort at a single institution. Second, the technical difficulty of portal venous bolus tracking in the presence of respiratory motion probably caused the wide variability of scan timing observed when SMV tracking was used. Third, we did not evaluate the diagnostic performance for livers with diseases such as tumors, fatty liver, or cirrhosis. Furthermore, in patients with cirrhosis accompanied by portal hypertension, time to the portal inflow of contrast material may well be variably delayed and portal venous enhancement reduced, which may affect the feasibility of portal venous bolus tracking.

\section{Conclusion}

Intense hepatic enhancement during the portal venous phase was robustly achieved using aortic bolus tracking in patients without chronic liver disease. Our results showed that portal-inflow bolus tracking was ineffective for increasing hepatic enhancement, reducing enhancement variability or improving image quality.

\section{REFERENCES}

[1] J. P. Heiken, J. A. Brink, B. L. McClennan, S. S. Sagel, H. P. Forman and J. DiCroce, "Dynamic Contrast-Enhanced CT of the Liver: Comparison of Contrast Medium Injection Rates and Uniphasic and Biphasic Injection Protocols," Radiology, Vol. 187, No. 2, 1993, pp. 327-331.

[2] Y. Yamashita, I. Ogata, J. Urata and M. Takahashi, "Cavernous Hemangioma of the Liver: Pathologic Correlation with Dynamic CT Findings," Radiology, Vol. 203, No. 1, 1997, pp. 121-125.

[3] J. F. Platt, I. R. Francis, J. H. Ellis and K. A. Reige,
"Liver Metastases: Early Detection Based on Abnormal Contrast Material Enhancement at Dual-Phase Helical CT,” Radiology, Vol. 205, No. 1, 1997, pp. 49-53.

[4] J. H. Oliver 3rd, R. L. Baron, M. P. Federle, B. C. Jones and R. Sheng, "Hypervascular Liver Metastases: Do Unenhanced and Hepatic Arterial Phase CT Images Affect Tumor Detection?” Radiology, Vol. 205, No. 3, 1997, pp. 709-715.

[5] E. K. Paulson, V. G. McDermott, M. T. Keogan, D. M. DeLong, M. G. Frederick and R. C. Nelson, "Carcinoid Metastases to the Liver: Role of Triple-Phase Helical CT," Radiology, Vol. 206, No. 1, 1998, pp. 143-150.

[6] L. Kopka, J. Rodenwaldt, U. Fischer, D. W. Mueller, J. W. Oestmann and E. Grabbe, "Dual-Phase Helical CT of the Liver: Effects of Bolus Tracking and Different Volumes of Contrast Material," Radiology, Vol. 201, No. 2, 1996, pp. 321-326.

[7] L. van Hoe, G. Marchal, A. L. Baert, S. Gryspeerdt and L. Mertens, "Determination of Scan Delay Time in Spiral CT-Angiography: Utility of a Test Bolus Injection," Journal of Computer Assisted Tomography, Vol. 19, No. 2, 1995 , pp. $216-220$.

doi:10.1097/00004728-199503000-00009

[8] M. Kanematsu, S. Goshima, H. Kondo, H. Nishibori, H. Kato, R. Yokoyama, T. Miyoshi, H. Hoshi, M. Onozuka and N. Moriyama, "Optimizing Scan Delays of Fixed Duration Contrast Injection in Contrast-Enhanced Biphasic Multidetector-Row CT for the Liver and the Detection of Hypervascular Hepatocellular Carcinoma," Jour- nal of Computer Assisted Tomography, Vol. 29, No. 2, 2005, pp. 195-201. doi:10.1097/01.rct.0000155062.50236.59

[9] F. A. Burgener and D. J. Hamlin, "Contrast Enhancement of Focal Hepatic Lesions in CT: Effect of Size and Histology," American Journal of Roentgenology, Vol. 140, No. 2, 1983, pp. 297-301. doi:10.2214/ajr.140.2.297

[10] K. Hanafusa, I. Ohashi, Y. Himeno, S. Suzuki and H. Shibuya, "Hepatic Hemangioma: Findings with TwoPhase CT," Radiology, Vol. 196, No. 2, 1995, pp. 465469.

[11] J. P. Heiken, J. A. Brink, B. L. McClennan, S. S. Sagel, T. M. Crowe and M. V. Gaines, "Dynamic Incremental CT: Effect of Volume and Concentration of Contrast Material and Patient Weight on Hepatic Enhancement," Radiology, Vol. 195, No. 2, 1995, pp. 353-357. 